\title{
Estimating overall persistence and long-range transport potential of persistent organic pollutants: a comparison of seven multimedia mass balance models and atmospheric transport models $\dagger$
}

\author{
A. Hollander, ${ }^{a b}$ M. Scheringer, ${ }^{* c}$ V. Shatalov, ${ }^{d}$ E. Mantseva, ${ }^{d}$ A. Sweetman, ${ }^{e}$ M. Roemer,${ }^{f}$ A. Baart,${ }^{g}$ \\ N. Suzuki, ${ }^{h}$ F. Wegmann ${ }^{c}$ and D. van de Meent ${ }^{a b}$ \\ Received 17th March 2008, Accepted 24th July 2008 \\ First published as an Advance Article on the web 12th August 2008 \\ DOI: 10.1039/b803760d
}

\begin{abstract}
Two different approaches to modeling the environmental fate of organic chemicals have been developed in recent years. The first approach is applied in multimedia box models, calculating average concentrations in homogeneous boxes which represent the different environmental media, based on intermedia partitioning, transport, and degradation processes. In the second approach, used in atmospheric transport models, the spatially and temporally variable atmospheric dynamics form the basis for calculating the environmental distribution of chemicals, from which also exchange processes to other environmental media are modeled. The main goal of the present study was to investigate if the multimedia mass balance models CliMoChem, SimpleBox, EVn-BETR, G-CIEMS, OECD Tool and the atmospheric transport models MSCE-POP and ADEPT predict the same rankings of the overall persistence $\left(P_{\mathrm{ov}}\right)$ and long-range transport potential (LRTP) of POPs, and to explain differences and similarities between the rankings by the mass distributions and inter-compartment mass flows. The study was performed for a group of 14 reference chemicals. For $P_{\mathrm{ov}}$, the models yield consistent results, owing to the large influence of phase partitioning parameters and degradation rate constants, which are used similarly by all models. Concerning LRTP, there are larger differences between the models than for $P_{\mathrm{ov}}$, due to different LRTP calculation methods and spatial model resolutions. Between atmospheric transport models and multimedia fate models, no large differences in mass distributions and inter-compartment flows can be recognized. Deviations in mass flows are mainly caused by the geometrical design of the models.
\end{abstract}

\section{Introduction}

Pollution caused by Persistent Organic Pollutants (POPs) is one of the large global environmental problems, due to the long environmental persistence of these substances and their ability to be transported over long distances. Historically, two different

${ }^{a}$ National Institute for Public Health and the Environment, Laboratory for Ecological Risk Assessment, P.O. Box 1, 3720 BA Bilthoven, The Netherlands

${ }^{b}$ Radboud University Nijmegen, Department of Environmental Science, P.O. Box 9010, 6500 GL Nijmegen, The Netherlands

'Swiss Federal Institute of Technology, ETH Hönggerberg, 8093 Zürich, Switzerland. E-mail: scheringer@chem.ethz.ch; Fax: +41-44-632 11 89; Tel: $+41-44-6323062$

${ }^{d}$ Meteorological Synthesizing Center East, Krasina pereulok, 16/1, 123056 Moscow, Russia

${ }^{e}$ Lancaster University, Environmental Science Department, LA1 4YQ Lancaster, United Kingdom

${ }^{f}$ TNO, Princetonplein 6, PO Box 80015, 3508 TA Utrecht, The Netherlands

${ }^{g}$ Delft Hydraulics, Rotterdamseweg 185, 2629 HD Delft, The Netherlands ${ }^{h}$ National Institute for Environmental Studies, 16-2 Onogawa, Tsukuba, Ibaraki 305-8506, Japan

$\dagger$ Electronic supplementary information (ESI) available: Chemical property data for the 14 reference chemicals; descriptions of the seven models; statistics of $P_{\text {ov }}$ and LRTP rankings of the 14 reference chemicals; mass balance estimates for PCB-153 in the year 2000. See DOI: $10.1039 / b 803760 d$ policy fields have been involved with POPs in the environment, namely air quality and chemical safety, and within each field an international convention on POPs was formulated. First, under the auspices of the United Nations Economic Commission for Europe (UNECE), the international community on air quality recognized the potential hazards of POPs, and within the framework of the Convention on Long-range Transboundary Air Pollution $^{1,2} 27$ countries (as of 2007) ratified the Protocol on Persistent Organic Pollutants. ${ }^{3}$ This Protocol encourages research, emission reduction and monitoring of POPs, as well as international co-operation between scientists. Second, the United Nations Environment Programme, ${ }^{4}$ which focuses on chemical safety, described its policy on POPs in the Stockholm Convention. ${ }^{5}$ In both fields, POP fate modeling is a relevant source of information. Mathematical models are increasingly used to simulate the environmental distribution of POPs, ${ }^{6-8}$ because there is only a limited amount of measurements available with a poor spatial and temporal coverage. Accordingly, the Executive Body of the CLRTAP, under its Cooperative Programme for Monitoring and Evaluation of the Long-range Transmission of Air Pollutants in Europe, initiated an intercomparison study between different POP fate models, to which experts of different countries would contribute. ${ }^{9}$ The material presented in this paper is a result of a part of this model intercomparison study. 
Two different POP fate modeling approaches have been developed in the recent years. ${ }^{8}$ The first is the multimedia partitioning approach, which is generally applied in multimedia box or mass balance models. This type of model assumes homogeneous environmental compartments, in which average concentrations are calculated based on advective and diffusive intermedia exchange processes (wet and dry deposition, volatilization, etc.), advective or macro-diffusive exchange between different geographical regions, and degradation. Due to their relatively low complexity, these models are often used in risk assessments, i.e. for screening assessments of large numbers of chemicals. ${ }^{10}$ In recent years, also spatially explicit multimedia mass balance models have been developed, e.g. IMPACT-2002, ${ }^{11}$ BETR-Global, ${ }^{12}$ G-CIEMS. ${ }^{13}$ The second approach, developed from the air quality field, is based on atmospheric dynamics and applied in atmospheric transport models. ${ }^{14,15}$ These models provide a higher temporal and spatial resolution than box models; they use a dynamic description of air flows based on mass, energy, and momentum conservation equations that are discretized over connected atmospheric volumes; to this description of air flow, several reservoirs representing the surface compartments are linked.

Both types of models can be used for the same goal: to identify chemicals with POP-like persistence and long-range transport potential. Ideally, both types of models would yield the same outcomes for descriptors of overall persistence $\left(P_{\text {ov }}\right)$ and longrange transport potential (LRTP). However, only few studies have investigated this question, ${ }^{\mathbf{8 , 1 6}}$ and it is not sufficiently clear how consistent the different models are. First, the different spatial and temporal resolutions used may lead to differences in model predictions. Second, different models of the same type may predict different concentrations and process intensities owing to variation in model algorithms and geometric dimensions. The following aspects have been addressed in recent model comparison studies: a number of spatially explicit models have been compared with non-spatial versions of the same model domain. ${ }^{11,17,18}$ Wania and Dugani ${ }^{19}$ compared the LRTP estimates of polybrominated diphenyl ethers for four multimedia fate models. Fenner et al. ${ }^{16}$ performed a model inter-comparison study on the predicted $P_{\text {ov }}$ and LRTP of chemicals for nine multimedia fate models. However, these studies did not consider atmospheric transport models. Hansen et $a .^{8}$ explored the differences in predicted concentrations of $\alpha$-hexachlorocyclohexane $(\alpha-\mathrm{HCH})$ between an atmospheric transport model and a multimedia mass balance model. They compared EVn-BETR $^{20,21}$ with DEHM-POP, ${ }^{22}$ and related the predicted concentration differences to the differences in the model description of environmental processes in the two models. Hollander et al. ${ }^{23}$ compared average concentrations and spatial concentration patterns of PCB-153 between the atmospheric model LOTOS-EUROS and the multimedia mass balance model SimpleBox. In these two studies, only one single multimedia model and one atmospheric model were taken into account, which makes it difficult to make general statements on the performance of the two model types. Lammel et al. ${ }^{24}$ presented a comparison between the multimedia models SimpleBox 2.0, ${ }^{25}$ Chemrange $1.0,{ }^{26,27} \mathrm{MPI}-\mathrm{MBM}^{28}$ and the atmospheric transport model MCTM ${ }^{15,29,30}$ for six substances. Although they found deviations between the models for the quantification of the
LRTP of these compounds, they concluded that there is good agreement between the models with respect to the LRTP-ranking of chemicals.

The present study goes beyond the previous investigations in that it includes more and/or more diverse models than the earlier studies. The main goal of the study is to investigate if the multimedia mass balance models CliMoChem, ${ }^{31}$ SimpleBox, ${ }^{32}$ EVn-BETR, ${ }^{20,21}$ G-CIEMS, ${ }^{13}$ OECD Tool ${ }^{33}$ and the atmospheric transport models $\mathrm{MSCE}-\mathrm{POP}^{7}$ and $\mathrm{ADEPT}^{34}$ yield similar rankings of the $P_{\text {ov }}$ and LRTP of selected organic chemicals. The second goal is to analyse the mass distributions and intercompartment mass flows of one selected chemical, PCB-153, and to discuss differences and similarities between the rankings in the light of the results obtained for PCB-153.

\section{Material and methods}

\section{Model setup}

Seven models for predicting the environmental fate of POPs participated in this inter-comparison study, of which two are derived from atmospheric transport models (ADEPT and MSCE-POP). The other five models are based on a multimedia mass balance modeling approach, i.e. EVnBETR, SimpleBox, G-CIEMS and CliMoChem, OECD Tool. The models differ considerably in their spatial resolutions. In the comparison of $P_{\text {ov }}$ and LRTP rankings, all models were reviewed, whereas the mass balance analysis was performed for five of seven models (not for ADEPT and the OECD Tool). A description of the models is given in the ESI, $\uparrow$ and a schematic presentation of their key features is given in Shatalov et al. ${ }^{35}$

The study was performed for the domain covering the area of $35^{\circ}-70^{\circ} \mathrm{N}$ and $10^{\circ} \mathrm{W}-30^{\circ} \mathrm{E}$, which represents Europe and some parts of North Africa and the North Atlantic and Arctic oceans. For this domain, data on land cover, leaf area indices and organic matter contents in the soil on a $1^{\circ} \times 1^{\circ}$ scale were used by all models. Land cover data were derived from the USGS Land Use/Land Cover dataset obtained from the NCAR Mesoscale Modeling System (MM5). ${ }^{36}$ The 25 specified categories of the original land cover database were aggregated to represent the land cover classes that are distinguished in each model (i.e. three classes in CliMoChem, six in MSCE-POP, five in SimpleBox). Leaf area indices were derived from Sellers et al. ${ }^{37}$ and organic matter contents in soil were obtained from NASA. ${ }^{38}$ In the models having a resolution coarser than $1^{\circ} \times 1^{\circ}$, the environmental data from the original dataset were averaged according to the models' actual resolution. The spatially explicit environmental input data were assumed to be constant in time during the calculation period. Beside these data, each model used its own additional environmental parameters. Information on the environmental parameters used by each individual model can be found in Shatalov et al. ${ }^{35}$

\section{$P_{\text {ov }}$ and LRTP estimates}

Each model was used to provide an estimate of $P_{\text {ov }}$ and LRTP for a group of 14 reference chemicals, and to derive rankings from high to low $P_{\mathrm{ov}}$ and LRTP. $P_{\mathrm{ov}}$ and LRTP are environmental hazard indicators that are often applied in chemicals assessment. The selected chemicals are aldrin, atrazine, 
benzo $[a]$ pyrene $(\mathrm{B}[a] \mathrm{P})$, brominated diphenyl ethers (BDE-47; BDE-99), biphenyl, carbon tetrachloride $\left(\mathrm{CCl}_{4}\right)$, hexachlorobenzene (HCB), hexachlorobutadiene (HCBD), $\alpha-\mathrm{HCH}$, p-cresol, and polychlorinated biphenyls (PCB-180; PCB-153; PCB-28). Their physical-chemical properties are given in the ESI. $\uparrow$ The emission scenario used in the $P_{\text {ov }}$ and LRTP calculations was a single pulse release to air located at $10^{\circ} \mathrm{E}$ and $52.5^{\circ}$ $\mathrm{N}$, followed by a no-emission simulation period of 20 years. For both indicators, $P_{\mathrm{ov}}$ and LRTP, there are different metrics, i.e. ways of quantifying $P_{\text {ov }}$ and LRTP. Each model used its own $P_{\text {ov }}$ and LRTP metrics, according to differences in the construction of the models, see Table 1. Although defined slightly different in the different models, $P_{\text {ov }}$ largely reflects the turn-over time of the chemicals in the chosen model system. $P_{\mathrm{ov}}$ was not calculated in ADEPT, because ADEPT does not account for chemical stored in the surface media. In the OECD Tool, LRTP was calculated with two different methods, see Table 1. Rank correlation coefficients between the rankings of the 14 chemicals obtained with the different models were calculated. ${ }^{39}$

\section{Mass balance estimates of PCB-153}

To obtain some information about the environmental processes that are implemented in the models and influence the $P_{\mathrm{ov}}$ and LRTP rankings, mass distributions and inter-compartment flows were investigated for one example compound. This analysis of mass balances was performed for PCB-153 because it is a 'multimedia chemical', which means that after emission, it is distributed among different environmental compartments. The physical-chemical input data of PCB-153 are given in Table 2. Yearly averaged PCB air emission data for the period 1981 to 2000 were derived from Breivik et al..$^{41}$ on a $1^{\circ} \times 1^{\circ}$ scale and converted to grid-format as described by Shatalov et $a l^{42}$ The models were run for the period 1981-2000 with time intervals of one month for SimpleBox, EVn-BETR, G-CIEMS and MSCEPOP, and three months for CliMoChem.

Masses and concentrations of PCB-153 were recorded at the end of the year 2000 for air, water, and soil. Mass flows between the compartments were calculated and similarities and discrepancies between the models were analysed. When possible, differences between the individual models were traced back to general differences between (spatially explicit) multimedia mass balance models and atmospheric transport models.

\section{Results}

\section{$P_{o v}$ and LRTP rankings of chemicals}

In Fig. 1, the relative rankings of the 14 selected chemicals according to their overall persistence are given for the six models. A value of 1 represents the lowest $P_{\text {ov }}$ and a value of 14 the highest $P_{\text {ov }}$.

There is agreement between the models with respect to the $P_{\mathrm{ov}}$ rankings to the extent that the individual models in most cases differ by only one or two scores from the average ranking. The largest deviations of individual compound rankings can be found for HCBD, PCB-153, and PCB-180 in EVn-BETR. The mean deviation from the average ranking is 0.69 . The correlations of the $P_{\mathrm{ov}}$ rankings among the individual models are given in Table 3. The lowest correlation coefficient has a value of 0.81 , which confirms the consistency of the $P_{\text {ov }}$ rankings. The mean deviation from the average ranks is smallest for MSCE-POP, G-CIEMS, and CliMoChem (0.43 scores). $p$-Cresol and $\mathrm{CCl}_{4}$ are the chemicals with the most consistent rankings by all models; HCBD received the most diverse scores in the different models (see statistics of $P_{\text {ov }}$ rankings in Table $\mathrm{S} 2$ in the ESI $\dagger$ ).

Fig. 2 shows the relative rankings of the 14 selected POPs according to their LRTP. There is less agreement between the models according to the LRTP rankings than according to the $P_{\text {ov }}$ rankings. For LRTP, the individual models at maximum differ by seven scores from the average ranking, whereas the mean deviation from the average ranking is 1.4. The correlations between the LRTP rankings of the individual models are given in Table 4. The lowest correlation coefficients are observed for CliMoChem because the LRTP metric used in CliMoChem is conceptually different from the other LRTP metrics, see the discussion section. If CliMoChem is excluded, the lowest correlation coefficient is 0.57 (ADEPT $v s$. EVn-BETR). The mean deviation from the average ranks is smallest for the OECD Tool (0.7 and 1.0 scores); CliMoChem and ADEPT show the largest deviations (2.4 and 2.0 scores, respectively). The low-LRTP chemicals aldrin, $p$-cresol and $\mathrm{BaP}$ exhibit the most consistent rankings among all models (mean deviation from average rank around 0.5 scores); PCB-180, PCB-28 and $\alpha-\mathrm{HCH}$ are ranked most differently by the different models (mean difference from average rank around 2 scores; see statistics of the LRTP rankings in Table S3 in the ESI†).

\section{Masses of PCB-153 and inter-compartment fluxes}

In Fig. 3, the mass flows of PCB-153 at the end of 2000 are given as percentage of the emission for MSCE-POP, CliMoChem, SimpleBox, EVn-BETR, and G-CIEMS. In the grey boxes, the mass fractions of the chemical in the air, water, vegetation, and soil are shown. The detailed mass balance estimates of all five models is given in the ESI. $\uparrow$ Absolute concentrations of PCB-153 in air range from $0.44 \mathrm{pg} \mathrm{m}^{-3}$ in CliMoChem to $7.5 \mathrm{pg} \mathrm{m}^{-3}$ in EVn-BETR, and the masses in 2000 range from $27 \mathrm{~kg}$ in CliMoChem to $107 \mathrm{~kg}$ in MSCE-POP. Concentrations in water range from $0.2 \mathrm{pg}^{-1}$ in G-CIEMS to $2.0 \mathrm{pg} \mathrm{l}^{-1}$ in SimpleBox, and soil concentrations lie between $12 \mathrm{pg} \mathrm{g}^{-1}$ in CliMoChem and $63 \mathrm{pg} \mathrm{g}^{-1}$ in G-CIEMS. MSCE-POP only predicts the concentration at the interface between soil and air, which for PCB-153 is $168 \mathrm{pg} \mathrm{g}^{-1}$ in 2000 . Fig. 3 shows that in all models nearly the total mass of PCB-153 is present in the soil compartment. Only a small fraction is present in air and vegetation, and $0.4 \%$ (G-CIEMS) to $3.6 \%$ (SimpleBox) resides in ocean water. The estimated mass flows of PCB-153 between the air, water and soil compartments, and the net export flows are more different for the different models than the relative mass fractions. Large differences between the models occur in the export flow with air and water out of the model domain. MSCE-POP and G-CIEMS estimate a high export flow from the air compartment $(63 \%$, resp. $52 \%$ of the emission of the year 2000 versus $12 \%-37 \%$ for the other models). In SimpleBox and EVn-BETR the export flow from the water compartment is relatively important $(22 \%-40 \%$ for SimpleBox and EVn-BETR versus $-0.3 \%$ to $7.6 \%$ for MSCE-POP, G-CIEMS and CliMoChem). The fraction degraded in water is relatively large in SimpleBox. 


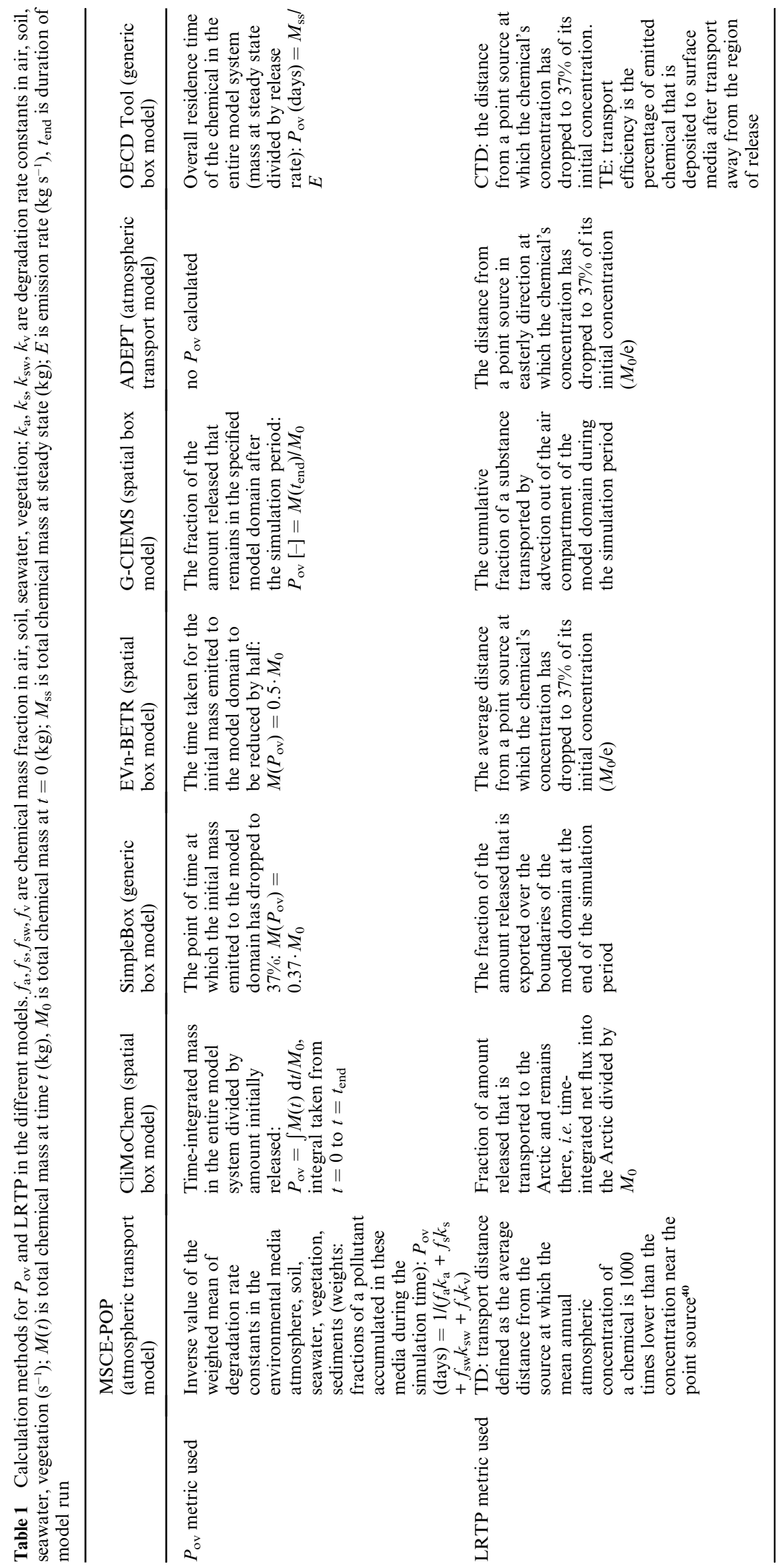


Table 2 Physical-chemical properties and degradation rate constants for PCB-153

\begin{tabular}{|c|c|c|}
\hline Parameter & Value & Unit \\
\hline Molecular weight ${ }^{43}$ & $3.61 \times 10^{+2}$ & $\mathrm{~g} \mathrm{~mol}^{-1}$ \\
\hline Vapor pressure at $25^{\circ} \mathrm{C}^{44}$ & $8.82 \times 10^{-5}$ & $\mathrm{~Pa}$ \\
\hline Water solubility at $25^{\circ} \mathrm{C}{ }^{44}$ & $6.50 \times 10^{-3}$ & $\mathrm{mg}^{-1}$ \\
\hline$K_{\text {Ow }} 44$ & $1.45 \times 10^{+7}$ & - \\
\hline $\begin{array}{l}\text { Gas/water partition } \\
\text { coefficient at } 25^{\circ} \mathrm{C}^{44}\end{array}$ & $2.09 \times 10^{-3}$ & - \\
\hline $\begin{array}{l}\text { Solids/water partition } \\
\text { coefficient at } 25^{\circ} \mathrm{C}^{44}\end{array}$ & $2.96 \times 10^{+5}$ & - \\
\hline Enthalpy of vaporization ${ }^{44}$ & $8.77 \times 10^{+1}$ & $\mathrm{~kJ} \mathrm{~mol}^{-1}$ \\
\hline Enthalpy of dissolution ${ }^{44}$ & $2.50 \times 10^{+1}$ & $\mathrm{~kJ} \mathrm{~mol}^{-1}$ \\
\hline $\begin{array}{l}\text { Gas phase degradation rate } \\
\text { constant at } 25^{\circ} \mathrm{C}^{43}\end{array}$ & $3.50 \times 10^{-8}$ & $\mathrm{~s}^{-1}$ \\
\hline $\begin{array}{l}\text { Dissolved phase } \\
\text { degradation rate constant at } \\
25^{\circ} \mathrm{C}^{43}\end{array}$ & $3.50 \times 10^{-9}$ & $\mathrm{~s}^{-1}$ \\
\hline $\begin{array}{l}\text { Bulk degradation rate } \\
\text { constant in sediment at } 25 \\
{ }^{\circ} \mathrm{C}^{43}\end{array}$ & $3.50 \times 10^{-9}$ & $\mathrm{~s}^{-1}$ \\
\hline $\begin{array}{l}\text { Bulk degradation rate } \\
\text { constant in soil at } 25^{\circ} \mathrm{C}^{43}\end{array}$ & $3.50 \times 10^{-9}$ & $\mathrm{~s}^{-1}$ \\
\hline
\end{tabular}

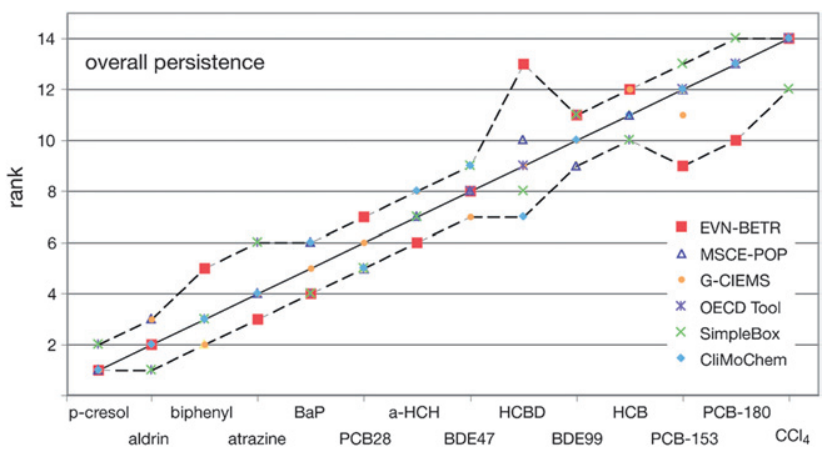

Fig. 1 Relative rankings of the 14 selected test chemicals according to their overall persistence $\left(P_{\mathrm{ov}}\right)$ for six models (ADEPT does not yield $P_{\mathrm{ov}}$ ). Values 1 and 14 represent the lowest and highest $P_{\mathrm{ov}}$, respectively. The solid line shows the average ranking of the chemicals in the six models. The dashed lines show the minimum and maximum rankings of the chemicals

Table 3 Rank correlations between the individual models according to their $P_{\mathrm{ov}}$ rankings of the 14 chemicals. The bottom line shows the correlation between each model and the average ranking of all models together

\begin{tabular}{|c|c|c|c|c|c|c|}
\hline & CliMoChem & $\begin{array}{l}\text { EVn- } \\
\text { BETR }\end{array}$ & $\begin{array}{l}\text { MSCE- } \\
\text { POP }\end{array}$ & SimpleBox & $\begin{array}{l}\text { OECD } \\
\text { Tool }\end{array}$ & $\begin{array}{l}\text { G- } \\
\text { CIEMS }\end{array}$ \\
\hline CliMoChem & 1.00 & 0.84 & 0.97 & 0.96 & 0.96 & 0.97 \\
\hline EVn-BETR & & 1.00 & 0.89 & 0.81 & 0.87 & 0.89 \\
\hline MSCE-POP & & & 1.00 & 0.93 & 0.96 & 0.98 \\
\hline SimpleBox & & & & 1.00 & 0.98 & 0.93 \\
\hline OECD Tool & & & & & 1.00 & 0.96 \\
\hline G-CIEMS & & & & & & 1.00 \\
\hline Average & 0.99 & 0.90 & 0.99 & 0.96 & 0.98 & 0.99 \\
\hline
\end{tabular}

Total deposition from air to surface media increases from MSCE-POP (37\%) to G-CIEMS (50\%), EVn-BETR (62\%), CliMoChem (79\%), and SimpleBox (87\%). In 2000, all models except MSCE-POP show a net deposition flow from air to soil,

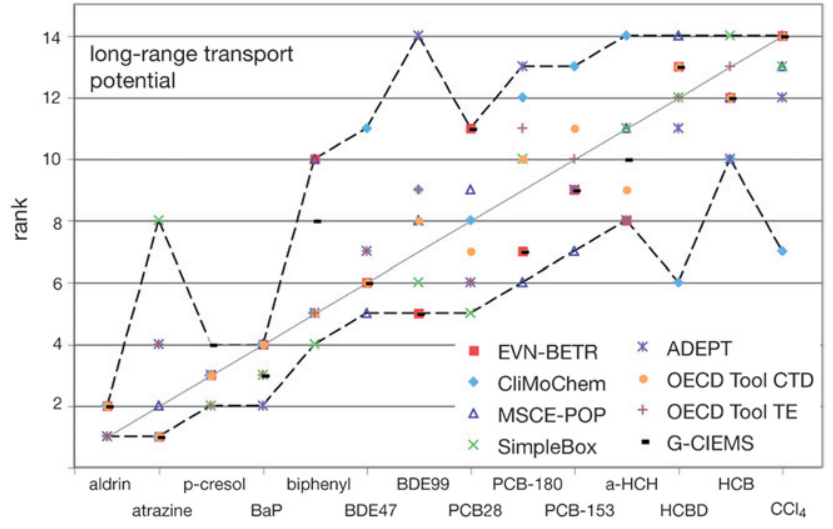

Fig. 2 Relative rankings of the 14 selected test chemicals according to their long-range transport potential (LRTP) for the seven models. The value 1 represents the lowest LRTP and the value 14 the highest LRTP. The solid line shows the average ranking of the chemicals in all models. The dashed lines show the minimum and maximum rankings of the chemicals.

with the largest flow for SimpleBox (40\%). In MSCE-POP, a net volatilization flow of $5.9 \%$ is found. Deposition to vegetation is largest for CliMoChem and MSCE-POP and, consequently, the mass flow from vegetation to soil is also relatively large in these models. In CliMoChem, a large fraction of the emitted PCB-153 is degraded in vegetation. MSCE-POP does not take into account this process in its model algorithm. In EVn-BETR, a relatively small fraction $(25 \%)$ of the emitted substance is degraded in the soil. MSCE-POP distinguishes degradation in the litter layer (10\% of the emitted PCB-153) and degradation in the soil ( $78 \%$ of the emissions).

\section{Discussion}

\section{$P_{\text {ov }}$ rankings of chemicals}

With respect to $P_{\text {ov }}$ the models are highly correlated, because degradation and phase partitioning are described similarly in all models. The half-lives of a chemical in a given environmental medium can be expected to be similar in all models, because the models use the same input values (valid for $298 \mathrm{~K}$ ) and adjust these values to temperatures different from $298 \mathrm{~K}$ in a similar way. In addition to the degradation half-lives, the distributions between air, water and soil influence $P_{\mathrm{ov}}$. This distribution depends on the partition coefficients of the chemical and on the relative sizes of the different environmental media, i.e. the model geometry. The good agreement for $P_{\mathrm{ov}}$ indicates that also phase partitioning is relatively similar in the models. Beside degradation and partition coefficients, another possible cause of differences between the $P_{\text {ov }}$-rankings is the way of calculating $P_{\text {ov }}$. The most essential difference between the calculation methods is that some methods only consider degradation as a process determining persistence (closed models, i.e. MSCE-POP, CliMoChem), whereas others also take into account advective removal from the model domain ('open models'; i.e. SimpleBox, G-CIEMS). However, this may affect mainly absolute $P_{\mathrm{ov}^{-}}$ values - with lower absolute values in open models - but if the open and closed models yield similar fractions in air, the higher 
Table 4 Rank correlations between the individual models according to their LRTP rankings of the 14 chemicals. The bottom line shows the correlation between each model and the average ranking of all models together

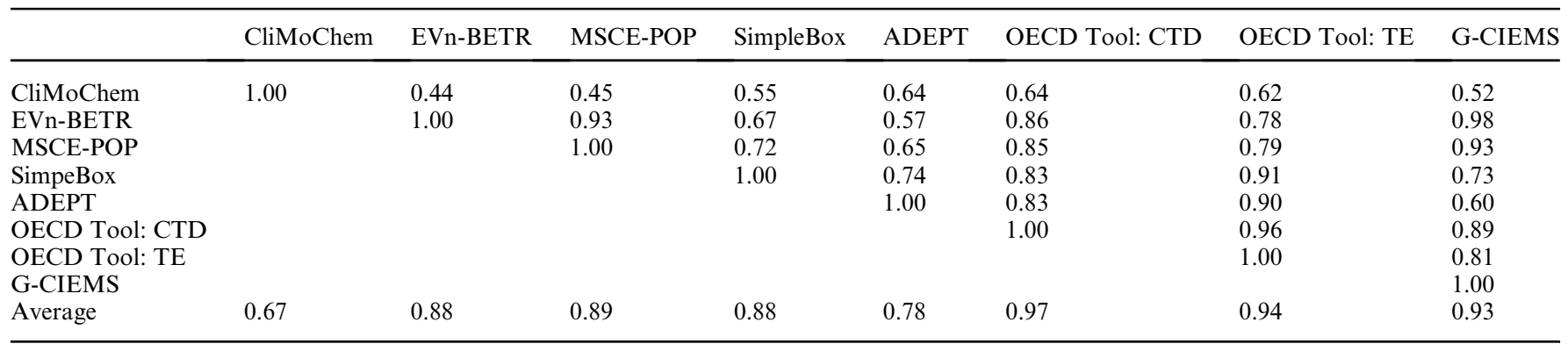

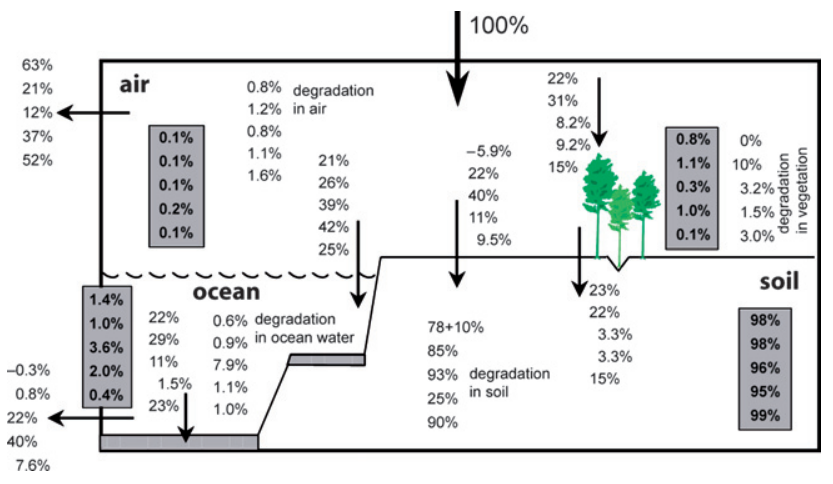

Fig. 3 Mass flows calculated for PCB-153 in 2000 as percentage of the emission to air. All losses from air add up to $100 \%$. The overall loss from the system (sum of all degradation and export fluxes) exceeds $100 \%$ because the models are not at steady state. In the grey boxes, mass fractions of PCB-153 in air, ocean water, vegetation and soil are displayed (except for rounding errors, these percentages add up to $100 \%$ ). From top to bottom, the numbers represent MSCE-POP, CliMoChem, SimpleBox, EVn-BETR, G-CIEMS.

removal rate constant in air in open models applies to all chemicals and does not strongly affect the ranking. Good agreement among models on their $P_{\text {ov }}$ estimates was also recognized by Fenner et al. ${ }^{16}$ for nine multimedia mass balance models and Lammel et al. $^{24}$ for the atmospheric transport model MCTM and the multimedia mass balance models SimpleBox 2.0, Chemrange 1.0 and MPI-MBM.

\section{LRTP rankings of chemicals}

Concerning LRTP, there are larger differences between the models than for $P_{\text {ov }}$. First, this is caused by differences in the LRTP metrics. This effect is most pronounced for CliMoChem: LRTP in CliMoChem is calculated as the fraction of chemical that reaches the Arctic during the simulation period and remains there. Therefore, volatile chemicals such as $\mathrm{CCl}_{4}$ and $\mathrm{HCB}$, which receive high LRTP scores in all other models, have low scores in CliMoChem because they reach the Arctic but only a small fraction is deposited there whereas a large fraction is transported out of the Arctic again. Heavy PCBs, on the other hand, receive higher LRTP scores in CliMoChem because they have a relatively high fraction that reaches the Arctic and remains there. Second, the influence of import/export fluxes, which are modeled differently in models with different spatial resolution, ${ }^{8}$ is larger on LRTP than on $P_{\text {ov }}$. In more highly resolved models, air flows reflect dynamic atmospheric conditions, including higher wind speeds and episodic transport events, whereas in box models the long-term average of the atmospheric dynamics is used, which evens out the effect of episodic transport events. This is illustrated by the high correlations between the more highly resolved models MSCE-POP, G-CIEMS, and EVn-BETR, on one hand, and between the box models SimpleBox and OECD Tool, on the other hand. Finally, besides the LRTP metrics used and the spatial resolution of a model, the presence of a deep ocean compartment, which acts as a sink for POPs, is important. Deposition of chemical to the deep ocean reduces the LRTP, whereas in absence of this process, the export flow out of the water compartment is higher. In general, model geometry thus influences the LRTP estimates and rankings more strongly than the $P_{\text {ov }}$ rankings. Finally, these outcomes are only valid for a scenario with emissions to air. Emissions to soil or water would probably yield different results for the LRTP of chemicals, due to a shift in the mass balances and the relative importance of certain transport processes in the different scenarios.

\section{Masses of PCB-153 and inter-compartment mass fluxes}

The five models considered in the mass balance study for PCB153 in the EMEP region yield similar values for the mass fractions in air, water and soil. In all models, the soil is the main storage compartment for PCB-153 (fractions of 95\% or higher). During the emission period, a significant part of the substance was deposited onto the soil, and due to the low degradability of PCB-153, it accumulated there. During the last years of the calculation period, emissions were declining. As a result, MSCEPOP predicts a volatilization flux from soil to air in the year 2000 whereas in the other models, still a net deposition flux is calculated. Due to the large air-borne export flux of PCB-153 out of the model system that occurs in MSCE-POP if emissions are into air, the flux from soil to air is enhanced. Besides, MSCE-POP includes vertical resolution in soil concentrations, resulting in relatively high soil concentrations predicted in the upper soil layer.

The export of PCB-153 by air out of the considered domain is significantly lower in the box models SimpleBox and CliMoChem (12\% and 21\%) than in the spatially explicit models EVn-BETR, MSCE-POP and G-CIEMS (37\%, 63\%, and 52\%). Due to the higher spatial resolution, air concentrations in the 
border grid cells of the spatially explicit models are different from the average concentrations calculated by SimpleBox or CliMoChem. At the northern borders of the model domain, chemical concentrations in air are low, but at the southern and eastern borders, they are significantly higher than the average air concentrations from the total model area, resulting in a larger export flow of PCB-153 in the south and east. For EVn-BETR, the same phenomenon is observed, but due to the coarser model scale, the effect on the total export flow is less pronounced than for MSCE-POP. In EVn-BETR, export by air is by a factor of 1.5 to 2 lower than in MSCE-POP and G-CIEMS, but by a factor of three higher than in SimpleBox. A difference between atmospheric transport models and multimedia mass balance models in predicted transport flows in air was also found by Lammel et $a l .{ }^{24}$ who found a tendency of box models to overestimate particle deposition from air and to underestimate atmospheric transport velocity because box models do not represent the temporal and spatial variability of these parameters.

The third factor causing differences in the fraction of PCB-153 that is exported out of the open model domain is the atmospheric height assumed in the models. MSCE-POP uses an atmospheric height of $12000 \mathrm{~m}$, whereas SimpleBox only represents the lower $1000 \mathrm{~m}$ of the atmosphere. From the current study, it appears that the larger the modeled atmospheric height, the larger the export of PCB-153 via air. This is in agreement with Wania and Dugani, ${ }^{19}$ who concluded that a larger atmospheric mixing height strongly increases the predicted LRTP of PBDEs.

For CliMoChem, model geometry strongly influences the export flow in a fourth way. In this model, the considered region falls within two latitudinal zones of the model that have no boundaries in east and west directions, which means that only export in southward and northward directions is counted as net export in CliMoChem. The lack of east and west borders in CliMoChem also influences the absolute emissions, masses and concentrations of PCB-153; in CliMoChem, the emissions go into the entire latitudinal bands and the fraction of the emissions scaled to the EMEP domain is about six times lower than in the other models; the masses of PCB-153 found in the EMEP domain are two to 10 times lower.

All models indicate a net loss of PCB-153 between 1999 and 2000, accounting for 4000-5200 tonnes in MSCE-POP and SimpleBox and 445 tonnes in CliMoChem (see above; lower emissions and amounts because of instantaneous distribution in east-west direction). The net loss in EVn-BETR is lower than in the other models (except CliMoChem), caused by a relatively small amount of PCB-153 degraded in soil. This can be explained by the relatively high contribution of air and water advection as loss processes in EVn-BETR.

Export of PCB-153 from the model domain by water is relatively large in EVn-BETR and SimpleBox, and low in MSCEPOP and CliMoChem. On the other hand, in the latter models, a chemical flow from the surface ocean water to the deep ocean is included, which is lacking in the two former models. The sums of export and deep ocean flows in MSCE-POP and CliMoChem equal the export flows in EVn-BETR and SimpleBox, which explains the differences in the models when only export flows are compared.

The fraction of PCB-153 that is deposited to water is similar in all models (ranging from $21 \%$ to $42 \%$ of the amount released).
Deposition to vegetation is similar and relatively high in MSCEPOP and CliMoChem, whereas it is similar and relatively low in SimpleBox and EVn-BETR. These differences are caused by the assumed values of the mass transfer coefficient at the air/ vegetation interface. The total deposition flux from air is high in the multimedia box models (SimpleBox, EVn-BETR, CliMoChem) and clearly lower in the atmospheric model MSCEPOP. Precipitation is described as meteorology-driven events in MSCE-POP, whereas in the multimedia models, a constant precipitation flux is assumed, leading to higher estimation of the wet deposition flux in these models. These outcomes are in agreement with the comparison of DEHM-POP and EVn$\mathrm{BETR}^{8}$ and with Lammel et al. $^{24}$

\section{Multimedia mass balance models vs. atmospheric transport models}

From the analysis of the model differences and similarities, some conclusions on differences between atmospheric transport models and multimedia mass balance models, either spatially explicit or box models, can be drawn. Two dominant factors causing differences in model results are (i) differences in the way how chemical transport processes are modeled, and (ii) model geometry and spatial resolution. This second factor mainly influences export of substances out of the model domain by air transport, whereas differences in process descriptions (first factor) have the largest effect on the air-vegetation and air-soil exchange, as well as on the degradation rates in the water compartment.

Because spatial resolution is important for export mass fluxes and LRTP, the largest differences exist between non-spatial (box) models and spatially explicit models, independent of whether these are spatially resolved multimedia mass balance models or atmospheric transport models. At least for the scenario used in this study, differences in model algorithms and in descriptions of inter-compartment exchange processes are subordinate to whether the model is a gridded model or not. In general, this phenomenon will appear strongest in scenarios with a heterogeneous spatial emission pattern such as used here (one single point source, which is the most heterogeneous emission pattern that is possible), causing different estimates of export mass flows out of the model domain in the different types of models.

When choosing a model for analysing the environmental fate of POPs, either a spatial $v s$. non-spatial model or an atmospheric transport model vs. a multimedia mass balance model, one should take into account several factors. First, the environmental compartment(s) of interest are of importance. Chemical concentrations in air in an open domain are generally predicted more reliably by spatially explicit models due to their higher resolution, and even more by atmospheric transport models, due to their more accurate description of meteorological parameters and processes. ${ }^{8}$ For the less mobile media, particularly soils and sediments, the influence of model resolution on the calculated concentrations is much smaller. The second factor determining the choice of a spatial or non-spatial model is the varibility of emission intensities over the study area, since the emission pattern of compounds largely determines the concentration pattern. $^{11,45}$ If the emissions of a compound are highly 
heterogeneous within the study area, and the influence of this heterogeneity is to be evaluated by the model, a spatially explicit model has to be used to determine the environmental concentrations. In contrast, if the emissions are more evenly distributed or if one is only interested in average concentrations over a larger area, a non-spatial model is sufficient. In all cases, one should keep in mind that models are only simplified representations of reality, depending on the perspective of the model developer. Model choice thus should reflect on the goal of the study and the preferred accuracy in the outcomes. Whenever possible, the results should be compared to experimental data. Concerning the ranking of chemicals according to their overall persistence and long-range transport potential, there are only some minor differences between spatial and non-spatial models and between atmospheric transport models and multimedia mass balance models. These differences are mainly caused by differences in the calculation methods of $P_{\mathrm{ov}}$ and LRTP. The practical reason to choose multimedia mass balance models in chemical ranking exercises remains their simplicity of use. When large numbers of substances are to be processed, the use of more demanding atmospheric transport models may be impractical.

\section{References}

1 CLRTAP, Convention on Long-range Transboundary Air Pollution and its Protocols, United Nations Economic Commission for Europe, New York, 1979.

2 ECE/EB.AIR/50, Convention on Long-range Transboundary Air Pollution and its Protocols (CLRTAP), United Nations Economic Commission for Europe, New York, 1996.

3 ECE/EB.AIR/60, Protocol to the 1979 Convention on Long-range Transboundary Air Pollution on Persistent Organic Pollutants, United Nations, New York/Geneva, 1998.

4 UNEP, United Nations Environmental Programme, Decision 18/32. Persistent Organic Pollutants, United Nations Environmental Programme, division of Technology, Industry and Economics, 1995.

5 UNEP, United Nations Environmental Programme. Stockholm convention on persistent organic pollutants (POPs) - Text and Annexes, UNEP/Chemicals/2001/3, Geneva, Switzerland, 2001.

$6 \mathrm{M}$. Scheringer and F. Wania, Multimedia Models for Global Transport and Fate of Persistent Organic Pollutants, in Handbook of Environmental Chemistry, Vol. 3/O, Persistent Organic Pollutants ed. H. Fiedler and O. Hutzinger, Springer, Berlin/Heidelberg, 2003, pp. 237-269.

7 A. Gusev, E. Mantseva, V. Shatalov and B. Strukov, Regional Multicompartment Model MSCE-POP, EMEP/MSC-E Technical Report 5/2005, Meteorological Synthesizing Center East, Moscow, 2005.

8 K. M. Hansen, K. Prevedouros, A. Sweetman, K. C. Jones and J. H. Christensen, A Process-Oriented Inter-Comparison of a Box Model and an Atmospheric Chemistry Transport Model: Insights into Model Structure using $\alpha-\mathrm{HCH}$ as the Modelled Substance, Atmos. Environ., 2006, 40(12), 2089-2104.

9 ECE/EB.AIR/75, Report of the Nineteenth Session of the Executive Body for the Convention on Long Range Transboundary Air Pollution, United Nations Economic Commission for Europe, Geneva, Switzerland, 2002.

10 D. van de Meent and J. H. M. De Bruin, Risk Assessment of Chemicals: An Introduction, ed. C. J. van Leeuwen and T. G. Vermeire, Springer, Dordrecht, The Netherlands, 2007, pp. 159-193.

11 D. W. Pennington, M. Margni, C. Amman and O. Jolliet, Multimedia fate and human intake modeling: spatial versus nonspatial insights for chemical emissions in Western Europe, Environ. Sci. Technol., 2005, 39(4), 1119-1128.

12 M. MacLeod, W. J. Riley and T. E. McKone, Assessing the influence of climate variability on atmospheric concentrations of polychlorinated biphenyls using a global-scale mass balance model (BETR-Global), Environ. Sci. Technol., 2005, 39(17), 6749-6756.
13 N. Suzuki, K. Murasawa, T. Sakurai, K. Nansai, K. Matsuhashi, Y. Moriguchi, K. Tanabe, O. Nakasugi and M. Morita, Georeferenced multimedia environmental model (G-CIEMS): model formulation and comparison to the generic model and monitoring approaches, Environ. Sci. Technol., 2005, 38(21), 5682-5693.

14 A. Malanichev, E. Mantseva, V. Shatalov, B. Strukov and N. Vulykh, Numerical evaluation of the PCBs transport over the Northern Hemisphere, Environ. Pollut., 2004, 128(1-2), 279-289.

15 G. Lammel, J. Feichter and A. Leip, Long-range transport and global distribution of semivolatile organic compounds: A case study on two modern agrochemicals, Report No. 324, Max Planck Institute for Meteorology, Hamburg, Germany, 2001.

16 K. Fenner, M. Scheringer, M. MacLeod, M. Matthies, T. McKone, M. Stroebe, A. Beyer, M. Bonnell, A.-C. Le Gall, J. Klasmeier, D. Mackay, D. van de Meent, D. Pennington, B. Scharenberg, N. Suzuki and F. Wania, Comparing Estimates of Persistence and Long-Range Transport Potential among Multimedia Models, Environ. Sci. Technol., 2005, 39(7), 1932-1942.

17 J. M. Armitage, I. T. Cousins, M. Hauck, J. V. Harbers and M. A. J. Huijbregts, Empirical evaluation of spatial and non-spatial European-scale multimedia fate models: results an implications for chemical risk assessment, J. Environ. Monit., 2007, 9(6), 572-581.

18 O. Klepper and H. A. den Hollander, A comparison of spatially explicit and box models for the fate of chemicals in water, air and soil in Europe, Ecol. Modell., 1999, 116, 183-202.

19 F. Wania and C. B. Dugani, Assessing the long-range transport potential of polybrominated diphenyl ethers: a comparison for four multimedia models, Environ. Toxicol. Chem., 2003, 22, 12521261.

20 K. Prevedouros, M. MacLeod, K. C. Jones and A. J. Sweetman, Modelling the fate of persistent organic pollutants in Europe: Parameterisation of a gridded distribution model, Environ. Pollut., 2004, 128, 251-261.

21 K. Prevedouros, K. C. Jones and A. J. Sweetman, European-scale modeling of concentrations and distribution of polybrominated diphenyl ethers in the pentabromodiphenyl ether product, Environ. Sci. Technol., 2004, 38, 5993-6001.

22 K. M. Hansen, J. H. Christensen, J. Brandt, L. M. Frohn and C. Geels, Modelling atmospheric transport of $\alpha$-hexachlorocyclohexane in the Northern Hemisphere with a 3-D dynamical model: DEHM-POP, Atmos. Chem. Phys., 2004, 4, 1125-1137.

23 A. Hollander, F. Sauter, H. A. den Hollander, M. Huijbregts, A. Ragas and D. van de Meent, Spatial variance in multimedia mass balance models: comparison of LOTOS-EUROS and SimpleBox for PCB-153, Chemosphere, 2007, 68, 1318-1326.

24 G. Lammel, W. Klöpffer, V. S. Semeena, E. Schmidt and A. Leip, Multicompartmental fate of persistent substances. Comparison of predictions from multi-media box models and a multicompartment chemistry-atmospheric transport model, Environ. Sci. Pollut. Res., 2007, 14, 153-165.

25 L. J. Brandes, H. A. den Hollander and D. van de Meent, SimpleBox 2.0: a nested multimedia fate model for evaluating the environmental fate of chemicals, National Institute for Public Health and the Environment (RIVM), Report number 719101029, Bilthoven, The Netherlands, 1996.

26 M. Scheringer, Persistence and Spatial Range as Endpoints of an Exposure-Based Assessment of Organic Chemicals, Environ. Sci. Technol., 1996, 30(5), 1652-1659.

27 M. Scheringer, H. Held and M. Stroebe, Chemrange $2.1-A$ multimedia transport model for calculating persistence and spatial range of organic chemicals, Model and Software Description, ETH Zürich, 2002. Model available from www.sust-chem.ethz.ch/ research/product/chemrange.html.

28 G. Lammel, Effects of temporally averaging climate parameters on predicted multicompartmental fate of pesticides and POPs, Environ. Pollut., 2004, 128, 291-302.

29 V. S. Semeena and G. Lammel, Effects of various scenarios upon entry of DDT and $\gamma-\mathrm{HCH}$ into the global environment on their fate as predicted by a multicompartment chemistry-transport model, Fresenius Environ. Bull., 2003, 12, 925-939.

30 V. S. Semeena, J. Feichter and G. Lammel, Impact of the regional climate and substance properties on the fate and atmospheric longrange transport of persistent organic pollutants - Examples of DDT and $\gamma-\mathrm{HCH}$, Atmos. Chem. Phys. Discuss., 2005, 5, 12569 12615 . 
31 M. Scheringer, F. Wegmann, K. Fenner and K. Hungerbuhler, Investigation of the cold condensation of persistent organic pollutants with a global multimedia fate model, Environ. Sci. Technol., 2000, 34, 1842-1850.

32 H. A. den Hollander, J. C. H. van Eijkeren and D. van de Meent, SimpleBox 3.0: multimedia mass balance model for evaluating the fate of chemicals in the environment, National Institute for Public Health and the Environment (RIVM), Report number 601200003, Bilthoven, The Netherlands, 2004.

33 F. Wegmann, L. Cavin, M. MacLeod, M. Scheringer and K. Hungerbühler, The OECD software tool for screening chemicals for persistence and long-range transport potential, Environ. Modell. Software, 2008, DOI: 10.1016/j.envsoft.2008.06.014. Model available from www.oecd.org/LongAbstract/0,3425en_2649_34365_ 40718985_119669_1_1_1,00.html.

34 M. Roemer, A. Baart and J. M. Libre, ADEPT - Developent of an Atmospheric DEPosition and Transport model for risk assessment, TNO report B\&O-A R2005/208, Apeldoorn, The Netherlands, 2004

35 V. Shatalov, E. Mantseva, A. Baart, P. Bartlett, K. Breivik, J. Christensen, S. Dutchak, D. Kalweitt, R. Farrett, M. Fedyunin, S. Gong, K. M. Hansen, I. Holoubek, P. Huang, K. Jones, M. Matthies, G. Petersen, K. Prevedouros, J. Pudykiewicz, M. Roemer, M. Salzman, M. Scheringer, J. Stocker, B. Strukov, N. Suzuki, A. Sweetman, D. van de Meent and F. Wegmann, POP Model Intercomparison Study. Stage I: comparison of description of main processes determining POP behavior in various environmental compartments, EMEP/MSC-E Technical Report 1/2004, Meteorological Synthesizing Centre-East, Moscow, 2004.

36 Y. R. Guo and S. Chen, Terrain and Land Use for the Fifth-Generation Penn State/NCAR Mesoscale Modeling System (MM5): Program TERRAIN, NCAR Technical Note: NCAR/TN -397 + IA, Boulder, CO, 1994.

37 P. J. Sellers, B. W. Meeson, F. G. Hall, G. Asrar, R. E. Murphy, R. A. Schiffer, F. P. Bretherton, R. E. Dickinson, R. G. Ellingson, C. B. Field, K. F. Huemmrich, C. O. Justice, J. M. Melack,
N. T. Roulet, D. S. Schimel and P. D. Try, Remote sensing of the land surface for studies of global change: Models - algorithmsexperiments, Remote Sens. Environ., 1994, 51, 3-26.

38 NASA, 2004, http://www.giss.nasa.gov (visited on March 2nd, 2004).

39 R. V. Hogg and A. T. Craig, Introduction to Mathematical Statistics, 5th edn, Macmillan, New York, 1995, p. 338.

40 B. D. Rodan, N. Eckley, D. W. Pennington and R. S. Boethling, Screening persistent organic pollutants: techniques to provide a scientific basis for POPs criteria in international negotiations, Environ. Sci. Technol., 1999, 33, 3482-3488.

41 K. Breivik, A. Sweetman, J. M. Pacyna and K. C. Jones, Towards a global historical emission inventory for selected PCB congeners a mass balance approach, 2. Emissions, Sci. Tot. Environ., 2002, 290, 199-224.

42 V. Shatalov, E. Mantseva, A. Baart, P. Bartlett, K. Brevik, J. Christensen, S. Dutchak, S. Gong, A. Gusev, K. M. Hansen, A. Hollander, P. Huang, K. Hungerbühler, K. Jones, G. Petersen, M. Roemer, M. Scheringer, J. Stocker, N. Suzuki, A. Sweetman, D. van de Meent and F. Wegmann, POP model intercomparison study. Stage II: comparison of mass balance estimates and sensitivity studies, EMEP/MSC-E Technical Report 5/2006, Meteorological Synthesizing Centre-East, Moscow, 2006.

43 D. Mackay, W. Y. Shiu and K. C. Ma, Illustrated handbook of physical-chemical properties and environmental fate for organic chemicals. Volume I: Monoaromatic hydrocarbons, chlorobenzenes, and PCBs, Lewis Publishers, New York, 1992.

44 N. Li, F. Wania, D. Y. Lei and G. L. Daly, A comprehensive and critical compilation, evaluation and selection of physical chemical property data for selected polychlorinated biphenyls, J. Phys. Chem. Ref. Data, 2003, 32, 1545-1590.

45 A. Sweetman, I. T. Cousins, R. Seth, K. C. Jones and D. Mackay, A dynamic level IV multimedia environmental model: Application to the fate of polychlorinated biphenyls in the United Kingdom over a 60-year period, Environ. Toxicol. Chem., 2002, 21, 931-940. 